\title{
Formation of superfluid liquid pocket in aerogel and its solidification by cooling
}

\author{
H. Matsuda, A. Ochi, R. Isozaki, R. Nomura, and Y. Okuda \\ Department of Physics, Tokyo Institute of Technology, 2-12-1 O-okayama, Meguro, Tokyo 152-8551, Japan \\ E-mail: nomura.r.aa@m.titech.ac.jp
}

Received February 25, 2013

\begin{abstract}
Formation of superfluid liquid pockets of ${ }^{4} \mathrm{He}$ surrounded by ${ }^{4} \mathrm{He}$ crystals were observed in an aerogel of $96 \%$ porosity. The liquid pockets did not crystallize by application of pressure but crystallized via avalanche by cooling below a particular temperature. The crystallization by cooling was also observed when crystals occupied a smaller portion of the aerogel. Driving force for the crystallization by cooling and possible mass transport process are discussed.
\end{abstract}

PACS: 64.70.D- Solid-liquid transitions;

67.80.bf Liquid-solid interfaces; growth kinetics;

67.25.D- Superfluid phase.

Keywords: superfluid ${ }^{4} \mathrm{He}$, crystal growth, aerogel, supersolid.

\section{Introduction}

Properties of superfluid ${ }^{4} \mathrm{He}$ in porous materials have been investigated extensively and variations in the static properties, such as reduction in the superfluid transition temperatures and elevation of the crystallization pressure have been revealed [1-9]. However, not much is known about the dynamical process of superfluid ${ }^{4} \mathrm{He}$ in porous materials due to the lack of an appropriate method to tackle the physics. Silica-aerogels are highly porous materials whose pore size is on a nanometer scale while at the same time being very transparent; we can thus investigate the dynamics inside the material by visual observation. There have been several reports on the imbibition process of normal fluid and superfluid ${ }^{4} \mathrm{He}$ in aerogel by visualization [10-12]; superfluid ${ }^{4} \mathrm{He}$ is imbibed in aerogel at a constant rate while normal fluid ${ }^{4} \mathrm{He}$ is imbibed following the classical Washburn law. Anomalous two-step imbibition was also found in highly porous aerogels [11]. These observations can be regarded as early trials for the nanofluidics in both classical and quantum liquids.

Crystallization dynamics of superfluid ${ }^{4} \mathrm{He}$ has also been investigated visually in aerogel $[13,14]$. A variablevolume cell allowed us to make crystals at a constant temperature and the entire crystallization process was observed visually in the transparent aerogels. Crystallization of ${ }^{4} \mathrm{He}$ in aerogels shows a dynamical phase transition at around $600 \mathrm{mK}$ which is the result of the competition between thermal fluctuation and spatial disorder: crystals grow via creep at high temperatures and via avalanche at low tem- peratures [13]. The creep and avalanche growth was clarified as the thermal activation and macroscopic quantum tunneling, respectively, from the crystallization rate and nucleation probability measurements $[14,15]$. In the quantum growth regime, the avalanche size distribution followed a power law, demonstrating the self-organized criticality at low temperatures where the quantum process governs the dynamics [14,16].

In this paper, we report a formation of superfluid liquid pockets surrounded by crystals in one particular aerogel. The liquid pockets always appeared when the crystals were grown in the high-temperature creep region and did not crystallize further even with the application of higher pressure at a constant temperature. With cooling, they were found to crystallize below a particular temperature via avalanche. Crystallization by cooling was also observed when crystals occupied a smaller portion of the aerogel. Possible mechanism for the crystallization by cooling is discussed. Some results of this paper have been reported elsewhere [17].

\section{Experimental setups}

Silica-aerogel is a transparent porous material with a highly open structure which consists of nanometer scale silica particles [18-22]. It has been used to investigate the disorder effects on condensed phases of He [3,9,11,13,14, 22-26]. A silica-aerogel sample of $96 \%$ porosity was grown in situ in a flat glass tube by Panasonic Corporation. The tube is shown in Fig. 1 and had an inner space $1 \mathrm{~mm}$ deep, $8 \mathrm{~mm}$ wide and $10 \mathrm{~mm}$ high. There was no gap between the inner wall 


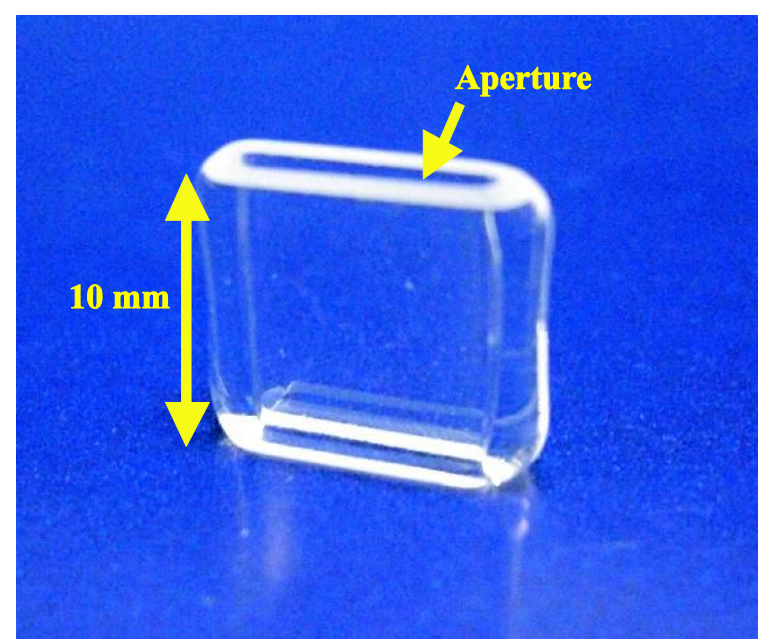

Fig. 1. (Color online) Flat glass tube to be filled with aerogel. Inner space of the tube is $1 \mathrm{~mm}$ deep, $8 \mathrm{~mm}$ wide and $10 \mathrm{~mm}$ high.

of the tube and the aerogel. The bottom of the tube was glued on a glass plate and the aerogel had contact with outer space only through the upper aperture.

The blocked capillary method was usually adopted to study crystallization of ${ }^{4} \mathrm{He}$ in porous material in earlier works. This temperature sweep method, however, made it difficult to study the details of the crystallization dynamics as a function of temperature. Instead, we decided to use a variable-volume cell which allows us to grow crystals in aerogel at a constant temperature. It has succeeded in revealing the temperature effect on the crystallization dynamics [13,14,27,28]. The variable-volume cell was composed of two chambers, a high-pressure chamber $(A)$ and a low-pressure chamber $(B)$. The aerogel was installed in chamber $A$. Part of both chambers was made of phosphor bronze bellows connected by a rigid copper rod. The volume of chamber $A$ was able to be controlled by the low-pressure liquid in chamber $B$ due to a larger cross section of the bellows for chamber $B$. Pressure of chamber $A$ was measured by a capacitive pressure gauge. Chamber $A$ has optical windows and its interior was observable from outside the cryostat. The cell was installed in a dilution refrigerator with optical access. The initial condensation of ${ }^{4} \mathrm{He}$ gas was finished above the critical point temperature to avoid damage due to shrinkage of the aerogel caused by the gas-liquid interface [29,30]. Detailed description of the sample cell was given in publications [13,14,27,28].

\section{Results \\ 3.1. Liquid pocket formation and its crystallization by cooling}

The initial condition of the experiment was such that ${ }^{4} \mathrm{He}$ bulk crystals and superfluid liquid coexisted in the space outside of the aerogel at the bulk crystallization pressure; only the superfluid liquid existed in the aerogel at this moment due to the elevated crystallization pressure in the aerogel. Next, we started pressurizing chamber $B$ continuously at a fixed rate in the high-temperature creep region. The outer bulk crystals grew with the compression of chamber $A$ and finally filled the space outside of the aerogel. Thereafter, the pressure in chamber $A$ increased above the bulk crystallization pressure with the compression of bulk crystals.

For this aerogel, the first crystal nucleated at a particular point in the aerogel about $3 \mathrm{~mm}$ below the aperture at a pressure typically 2 bar above the bulk crystallization pressure as in Fig. 2(b). The crystal-superfluid interface in the aerogel expanded smoothly as in Figs. 2(c)-(e) because it was in the creep region at the high temperature. It was found in this aerogel that the crystallization eventually stopped and liquid pockets remained even if the compression of the chamber continued: two small superfluid pockets surrounded by crystals were left over in the aerogel as indicated by two arrows in Fig. 2(f). In other aerogels of the same porosity and of 98 and $99.5 \%$ porosities reported in our previous publications, crystallization always continued until the crystals completely filled the aerogel. A pressure-temperature trace of this process is shown in Fig. 3. The crosses labeled by 2(a) and 2(f) indicate the points at which Figs. 2(a) and 2(f) were photograghed, respectively. An overpressure of about 40 mbar was applied in this run but the leftover liquid pockets did not crystallize.

The liquid pockets were reproducibly observed for this aerogel whenever the crystals were grown in the creep region. They did not crystallize even with an application of $100 \mathrm{mbar}$ overpressure for $20 \mathrm{~min}$. The size of the liquid pockets changed from time to time and tended to be smaller at faster crystallizations. The larger pocket on the left part of the aerogel in Fig. 2(f) was always formed but its size was only about half that at faster crystallization. The smaller pocket on the right sometimes was not formed. However, when the crystals were grown in the same way in the low-temperature avalanche region, crystallization was completed to fill the aerogel and no liquid pocket was formed.

We stopped the supply of liquid ${ }^{4} \mathrm{He}$ to chamber $B$ and the compression of the chamber $A$ when the liquid pockets were formed. Immediately we cooled the system shown by the trace in Fig. 3. An enlarged image of the larger liquid pocket is shown in Fig. 4. During this cooling, the measured pressure of chamber $A$ was nearly constant. At $390 \mathrm{mK}$, the liquid pockets began to crystallize via avalanche at the cross 4(a) in Fig. 3 whose image is shown in Fig. 4(a). It is not possible to see from Fig. 4 if it is via avalanche but this is obviously confirmed from the video clip. The crystallization continued with cooling as in Figs. 4(b)-(e). At $190 \mathrm{mK}$ or at the cross 4(f) in Fig. 3, the whole portion of the liquid pocket crystallized as shown in Fig. 4(f). As the avalanche growth took place below $600 \mathrm{mK}$ when the crystals were grown in 

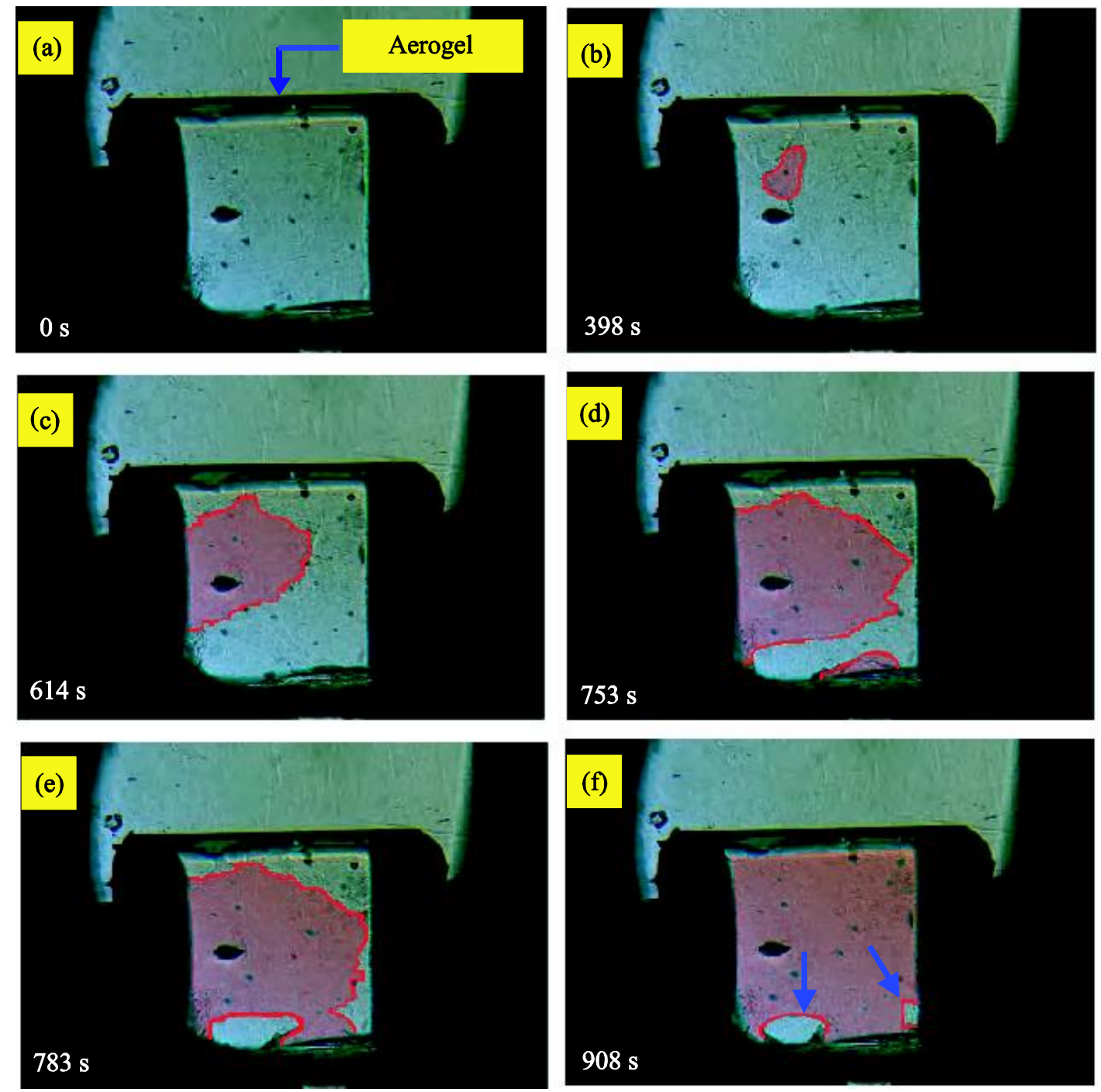

Fig. 2. (Color online) Crystallization image of ${ }^{4} \mathrm{He}$ via creep in a $96 \%$ porosity aerogel in a flat glass tube at $850 \mathrm{mK}$. Two superfluid liquid pockets were left unfrozen as indicated by arrows in (f). Time is indicated in each frame. The profile of the crystal-liquid interface is drawn and highlighted to aid clear viewing.

aerogel at a constant temperature, the liquid pockets grew via avalanche with the cooling below $390 \mathrm{mK}$.

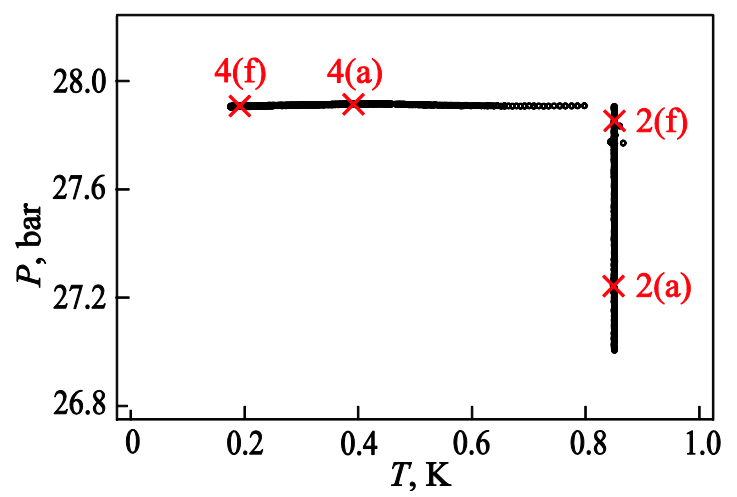

Fig. 3. (Color online) Temperature-pressure trace during the crystallization in Figs. 2 and 4. The crosses labeled by 2(a), 2(f), 4(a) and 4(f) are the points at which the photos of Figs. 2(a) and 2(f) and Figs. 4(a) and 4(f) were taken.

\subsection{Crystallization by cooling in a partly filled case}

After finding the crystallization of the liquid pocket, we decided to see what would happen by cooling when the crystallization was stopped before the formation of the liquid pocket in the same aerogel sample. The crystallization images and the pressure-temperature trace of this process are shown in Figs. 5 and 6. We first pressurized chamber $B$ continuously at a fixed rate to grow crystals in the aerogel in the high-temperature creep region as shown in Figs. 5(a) and 5(b), and stopped the crystallization when the crystals occupied a smaller portion, about one third of the aerogel as in Fig. 5(c). We photograghed Figs. 5(b) and 5(c) at the crosses indicated by (b) and (c) in Fig. 6. Contrary to the liquid pocket case, the bulk crystals still have contact with superfluid liquid in the aerogel. We waited at the constant temperature for about $750 \mathrm{~s}$ and the pressure dropped by about 30 mbar during this period; after that we cooled down the system. At $474 \mathrm{mK}$ the partially filled 

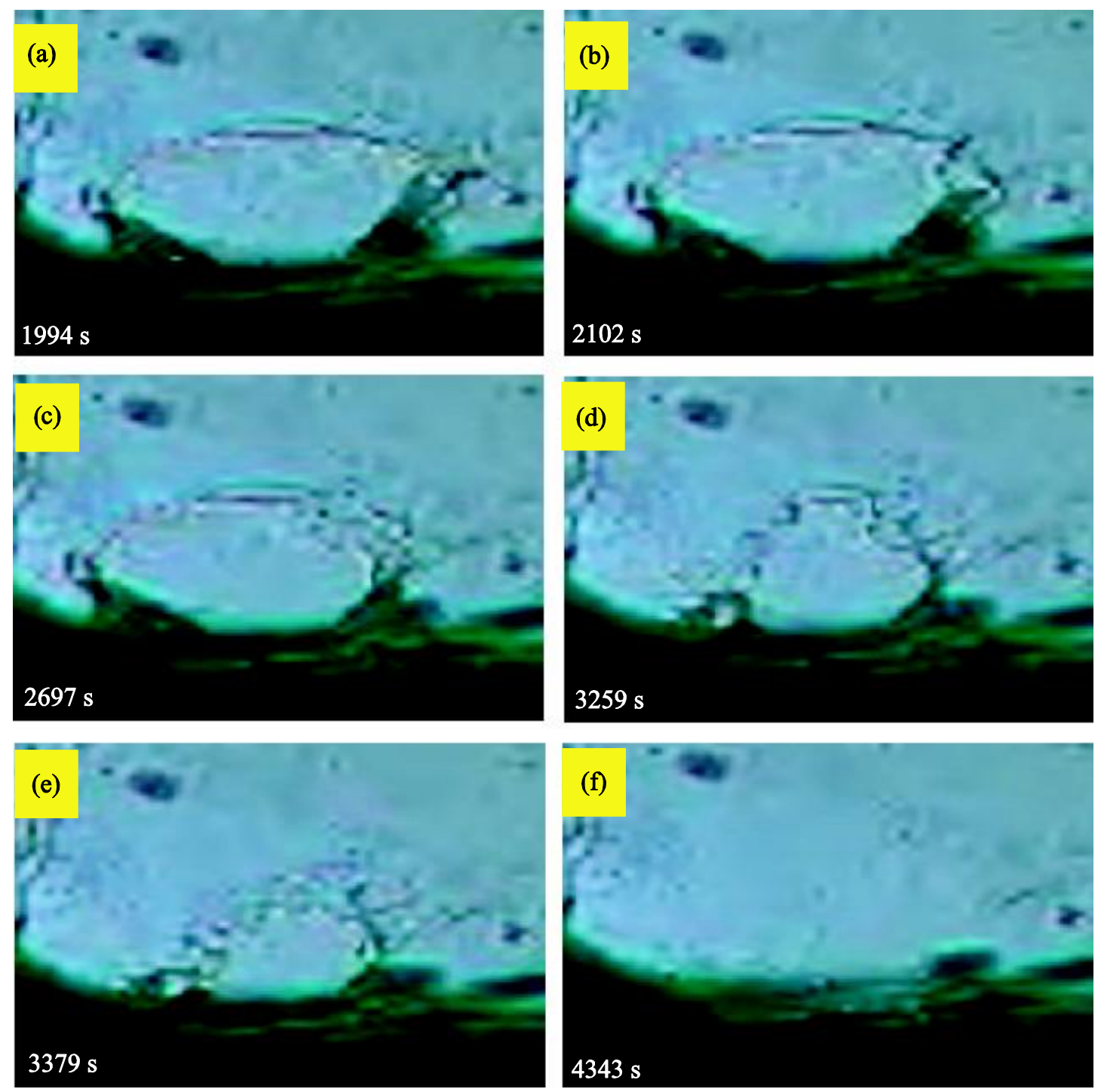

Fig. 4. (Color online) Enlarged image of crystallization of a superfluid liquid pocket in aerogel by cooling. Time is indicated in each frame.

crystals began to grow via avalanche as in Fig. 5(d) and continued to grow with further cooling as in Fig. 5(e). The crystallization temperature in the partially filled case was higher than that of the liquid pocket case. The crystallization by cooling did not fill the aerogel but eventually stopped as in Fig. 5(f). In Figs. 5(d)-(f), the profile of each avalanche is drawn for clarity. The crosses labeled by (d), (e) and (f) in Fig. 6 were the point at which Figs. 5(d)-(f) were photograghed.

\section{Discussion}

Due to the density difference between liquid and crystal, the mass transfer process has to be involved when crystals grow at constant pressure. From the observation that the crystals without any contact with outer bulk crystals grew far away from the aperture in the aerogel, it was concluded that the outer crystals were melted by the application of stress, ${ }^{4} \mathrm{He}$ atoms entered the aerogel in the superfluid state and recrystallized in the aerogel: the mass transport in aero- gel is through the superfluid flow and the possibility of the forced invasion of the outer crystals via plastic deformation was excluded as the mass transport mechanism [14]. Therefore, the crystallization should stop and liquid pockets should be left if a supply path of the superfluid is blocked by crystals in aerogel. Although the liquid pocket formation has been observed only in one aerogel reported in this paper, it offers another support for the assumed mass transport mechanism of crystallization in aerogel. In this aerogel, the initial nucleation point of the crystal happened to be closer to the upper opening than in other aerogels and it is reasonable that the supply pass was blocked. This may be the reason that the liquid pocket was observed only in this aerogel. In the avalanche region, a gap may exist between the crystals formed by the avalanche and act as the superfluid supply path for the crystallization to the end. In the other aerogels reported in the previous publications, the initial nucleation point was close to the bottom and away from the upper opening, and the crystal tended to grow from the bottom; the 

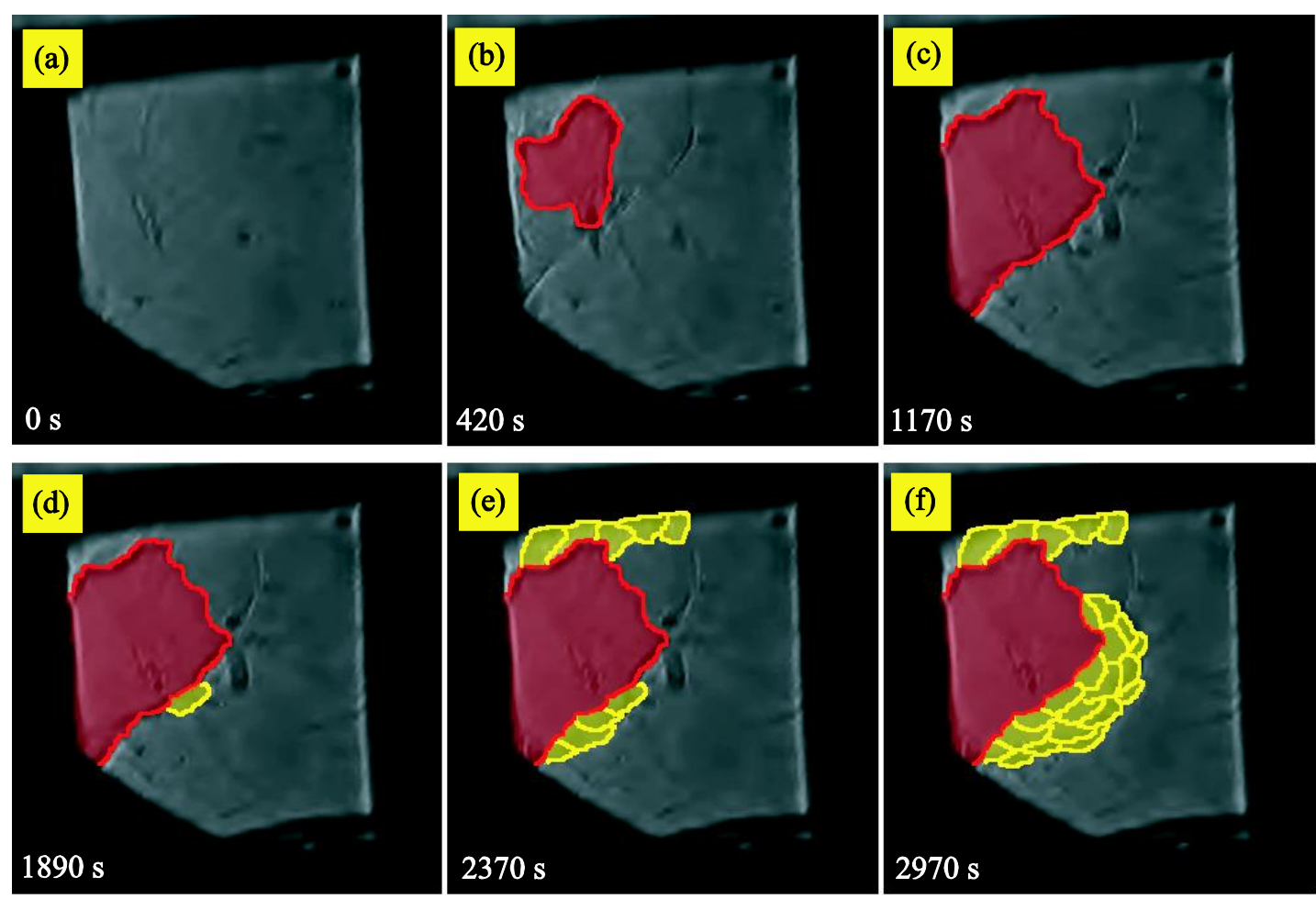

Fig. 5. (Color online) Crystallization image of ${ }^{4} \mathrm{He}$ in a $96 \%$ porosity aerogel in a flat glass tube. The crystals were grown at constant temperature as in (a), (b), and (c). Thereafter, the system was cooled down and the crystals began to grow via avalanche as in (d), (e), and (f). Time is indicated in each frame.

supply pass was not blocked and the crystallization was completed to the end.

The bulk crystallization pressure is nearly temperature independent at low temperatures and thus the driving force for the crystallization of ${ }^{4} \mathrm{He}$ is the overpressure from this bulk crystallization pressure $[31,32]$. What is the driving

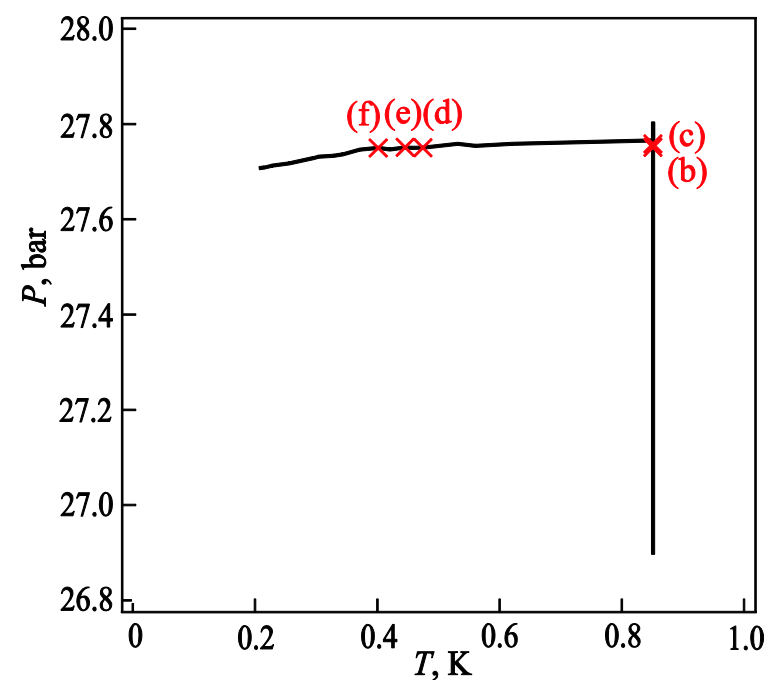

Fig. 6. (Color online) Temperature-pressure trace during the crystallization in Fig 5. The crosses labeled by (b)-(f) are the points at which the photos of Figs. 5(b)-(f) were taken. force for the crystallization by cooling in aerogel? In this aerogel the crystallization pressure at which the first crystal appears in aerogel was $(27.2 \pm 0.2)$ bar and no significant temperature dependence was observed in this highly porous material as in bulk. Therefore, the driving force for the crystallization in aerogel would also be the overpressure. This means that crystallization via cooling in aerogel is very anomalous because no additional driving force is gained by cooling.

Once the crystals undergo the supersolid transition, mass transport through them should become possible $[33,34]$. Therefore, one possible explanation for the crystallization by cooling could be related to the supersolid transition. The outer bulk crystals were stressed by bellows and were possibly sustaining a density gradient by elasticity; they would have a larger chemical potential than in aerogel. When the bulk crystals became supersolid below a transition temperature, mass transport through them should take place to equilibrate the system. In the partially filled case, ${ }^{4} \mathrm{He}$ atoms carried through the bulk crystals would be able to enter the aerogel as a superfluid liquid since the bulk crystals had direct contact with the superfluid liquid in it. Thereafter, ${ }^{4} \mathrm{He}$ atoms would be transferred through the aerogel and eventually recrystallize in the aerogel. Supersolidity of the bulk crystal would explain the crystallization by cooling in the partially filled case. 
In the liquid pocket case, however, supersolidity of the bulk crystals could not induce the crystallization; bulk crystal had no contact with the liquid pocket and the flow pass was blocked by the crystals in the aerogel. Crystals in the aerogel also needed to undergo the supersolid transition to carry the mass for the crystallization. The crystallization of the liquid pocket started at lower temperature than that of the partially filled case and this may be because the supersolid transition temperature was lower in the aerogel than in the bulk.

In order to determine whether the supersolidity is the case for the crystallization by cooling or different physics are taking place, more systematic measurements must be conducted; it will be informative to investigate the crystallization temperature by changing the overpressure after the formation of the liquid pocket or by changing the filling ratio of crystals in the aerogel. These measurements are in progress and hopefully will be reported in a future publication.

\section{Summary}

Making use of a variable-volume cell, crystals were grown in an $96 \%$ porosity aerogel at constant temperature in the creep growth region and it was found that some parts of the aerogel were left unfrozen as superfluid liquid pockets. The liquid pockets did not crystallize even if further pressure were applied to the system. This is because the superfluid path in the aerogel was blocked by crystals resulting in the cease of a mass supply for the crystallization. By cooling, however, the liquid pocket began to crystallize via avalanche below a particular temperature. This crystallization by cooling was also observed for crystals which partially filled the aerogel. The crystallization in the partially filled case began at higher temperature than in the liquid pocket. Possible scenario for the crystallization by cooling is the supersolidity of the crystals but further research is needed to come to a definite conclusion.

This study was supported in part by the Global Center of Excellence Program at Tokyo Tech. "Nanoscience and Quantum Physics Project", a Grant-in-Aid for Scientific Research (B) (Grant No. 21340095) from the Ministry of Education, Culture, Sports, Science and Technology of Japan and by a "Ground-based Research Announcement for Space Utilization” promoted by JAXA.

1. J.D. Reppy, Physica B+C 126, 335 (1984).

2. K. Shirahama, M. Kubota, S. Ogawa, N. Wada, and T. Watanabe, Phys. Rev. Lett. 64, 1541 (1990).

3. J. Yoon, D. Sergatskov, J. Ma, N. Mulders, and M.H.W. Chan, Phys. Rev. Lett. 80, 1461 (1998).

4. N. Wada, J. Taniguchi, H. Ikegami, S. Inagaki, and Y. Fukushima, Phys. Rev. Lett. 86, 4322 (2001).

5. K. Yamamoto, H. Nakashima, Y. Shibayama, and K. Shirahama, Phys. Rev. Lett. 93, 075302 (2004).

6. E.D. Adams, K. Uhlig, Y.-H. Tang, and G.E. Haas, Phys. Rev. Lett. 52, 2249 (1984).
7. J.R. Beamish, A. Hikata, L. Tell, and C. Elbaum, Phys. Rev. Lett. 50, 425 (1983).

8. K. Yamamoto, Y. Shibayama, and K. Shirahama, J. Phys. Soc. Jpn. 77, 013601 (2008).

9. K. Matsumoto, H. Tsuboya, K. Yoshino, S. Abe, H. Tsujii, and H. Suzuki, J. Phys. Soc. Jpn. 78, 034601 (2009).

10. W. Miyashita, K. Yoneyama, H. Kato, R. Nomura, and Y. Okuda, AIP Conf. Proc. 850, 251 (2006).

11. R. Nomura, W. Miyashita, K. Yoneyama, and Y. Okuda, Phys. Rev. E 73, 032601 (2006).

12. P. Spathis, A. Delga, C. Malheiro, and P.E. Wolf, J. Low Temp. Phys. 171, 693 (2013).

13. R. Nomura, A. Osawa, T. Mimori, K. Ueno, H. Kato, and Y. Okuda, Phys. Rev. Lett. 101, 175703 (2008).

14. R. Nomura, H. Matsuda, R. Masumoto, K. Ueno, and Y. Okuda, J. Phys. Soc. Jpn. 80, 123601 (2011).

15. H. Matsuda, A. Ochi, R. Isozaki, R. Masumoto, R. Nomura, and Y. Okuda, Phys. Rev. E 87, 030401(R) (2013).

16. Y. Sasaki, JPSJ Online-News and Comments, December 2, (2011). (http://jpsj.ipap.jp/news/jpsj-nc_98.html)

17. H. Matsuda, A. Ochi, R. Isozaki, R. Nomura, and Y. Okuda, J. Low Temp. Phys. 171, 295 (2013).

18. T.M. Haard, G. Gervais, R. Nomura, and W.P. Halperin, Physica B 284, 289 (2000).

19. Y.K. Akimov, Inst. Exp. Tech. 46, 287 (2003).

20. J.V. Porto and J.M. Parpia, Phys. Rev. B 59, 14583 (1999).

21. F. Detcheverry, E. Kierlik, M.L. Rosinberg, and G. Tarjus, Phys. Rev. E 68, 061504 (2003).

22. W.P. Halperin, H. Choi, J.P. Davis, and J. Pollanen, J. Phys. Soc. Jpn. 77, 111002 (2008).

23. T. Herman, J. Day, and J. Beamish, Phys. Rev. B 72, 184202 (2005).

24. T. Lambert, C. Gabay, L. Puech, and P.E. Wolf, J. Low Temp. Phys. 134, 293 (2004).

25. N. Mulders, J.T. West, M.H.W. Chan, C.N. Kodituwakku, C.A. Burns, and L.B. Lurio, Phys. Rev. Lett. 101, 165303 (2008).

26. P.E. Wolf, F. Bonnet, L. Guyon, T. Lambert, S. Perraud, L. Puech, B. Rousset, and P. Thibault, Eur. Phys. J. E 28, 183 (2009).

27. A. Osawa, H. Kato, T. Mimori, R. Nomura, and Y. Okuda, J. Low Temp. Phys. 150, 499 (2008).

28. K. Ueno, R. Masumoto, T. Mimori, A. Osawa, R. Nomura, and Y. Okuda, J. Low Temp. Phys. 158, 490 (2010).

29. T. Herman, J. Day, and J. Beamish, Phys. Rev. B 73, 094127 (2006).

30. H. Kato, W. Miyashita, R. Nomura, and Y. Okuda, J. Low Temp. Phys. 148, 621 (2007).

31. S. Balibar, H. Alles, and A.Y. Parshin, Rev. Mod. Phys. 77, 317 (2005).

32. Y. Okuda and R. Nomura, J. Phys. Soc. Jpn. 77, 111009 (2008).

33. M.W. Ray and R.B. Hallock, Phys. Rev. Lett. 100, 235301 (2008).

34. M.W. Ray and R.B. Hallock, Phys. Rev. B 79, 224302 (2009). 